Title : will be set by the publisher

Editors : will be set by the publisher

EAS Publications Series, Vol. ?, 2021

\title{
WHEN DOES THE INTERGALACTIC MEDIUM BECOME ENRICHED?
}

\author{
Benjamin D. Oppenheimer ${ }^{1}$, Romeel Davé ${ }^{1}$ and Kristian Finlator ${ }^{1}$
}

\begin{abstract}
We use cosmological hydrodynamic simulations including galactic feedback based on observations of local starbursts to find a self-consistent evolutionary model capable of fitting the observations of the intergalactic metallicity history as traced by C IV between $z=$ $6.0 \rightarrow 1.5$. Our main finding is that despite the relative invariance in the measurement of $\Omega(\mathrm{C} \mathrm{IV})$ as well as the column density and linewidth distributions over this range, continual feedback from star formationdriven winds are able to reproduce the observations, while an early enrichment scenario where a majority of the metals are injected into the IGM at $z>6$ is disfavored. The constancy of the C IV observations results from a rising IGM metallicity content balanced by a declining C IV ionization fraction due to a 1) decreasing physical densities, 2) increasing ionization background strength, and 3) metals becoming more shock-heated at lower redshift. Our models predict that $\sim 20 \times$ more metals are injected into the IGM between $z=6 \rightarrow 2$ than at $z>6$. We show that the median $\mathrm{C}$ IV absorber at $z=2$ traces metals injected 1 Gyr earlier indicating that the typical metals traced by C IV are neither from very early times nor from very recent feedback.
\end{abstract}

\section{Introduction}

Metals observed in the intergalactic medium (IGM) via quasar absorption line spectroscopy are thought to originate from some form of star formation-driven outflows as dynamical disruption is too inefficient (Aguirre et al. 2001). When and how metals are injected into the IGM are still a matter of debate. Observations of C IV in the IGM show surprisingly little evolution between $z \sim 5 \rightarrow 2$ (Songaila 2001, Schaye et al. 2003 \& Songaila 2005), despite this being the age of peak star formation. These observations motivate early enrichment scenarios by primeval galaxies and/or Populations-III stars at $z>6$ where physical distances are smaller and the gravitational potential wells are shallower.

\footnotetext{
${ }^{1}$ Steward Observatory, 933 N. Cherry Ave., Tucson, AZ, USA
} 
Title : will be set by the publisher

In Oppenheimer \& Davé (2006) (hereafter OD06), we explore a variety of galactic outflow models in GADGET-2 cosmological hydrodynamic simulations with the specific emphasis on modeling the $\mathrm{C}$ IV absorption observations between $z=6.0 \rightarrow 1.5$. We are able to reproduce the main $\mathrm{C}$ IV observations including the relative invariance of $\Omega(\mathrm{C}$ IV) by implementing galactic-scale outflows based on observed local starbursts (Martin 2005. Rupke et al. 2005). Only a narrow range of wind parameters using the physically-motivated momentum-driven wind scenario (Murray, Quartet, \& Thompson 2005) are able to enrich the IGM without overheating it at early times. Our models favor metal enrichment tied to concurrent star formation, as opposed to an early enrichment scenario with the majority of metals injected into the IGM before $z=6$. Observations supporting this include the ubiquitous outflows seen from star-forming galaxies at high-redshift galaxies (Pettini et al. 2001) and enhanced C IV and O VI observed around Lyman-break galaxies at $z \sim 2-3$ (Adelberger et al. 2003, 2005).

In these proceedings, we provide further arguments that the majority of metals in the IGM cannot be injected at very early times. Instead, we show the increasing IGM metallicity is balanced by an increasing $\mathrm{C}$ IV ionization correction resulting in observations of $\Omega(\mathrm{C}$ IV $)$ that remain relatively invariant from $z=6 \rightarrow 2$. We further explore the age of the metals in the IGM traced by C IV absorbers.

\section{Simulations}

We use the N-body+hydrodynamic code GADGET-2 (Springel 2005) with improvements including metal-line cooling and tunable superwind feedback as described in $\S 2$ of OD06. Briefly, the simulations we discuss here use an $\Omega=0.3 \Lambda \mathrm{CDM}$ cosmology in a $16 h^{-1} \mathrm{Mpc}$ box represented with $2 \times 256^{3}$ particles, giving a gas particle resolution of $3.9 \times 10^{6} M_{\odot} . \S 6$ of OD06 shows that this sufficiently resolves most C IV absorbers.

For simplicity, we focus on only two of the six outflow models of OD06, the "constant wind" (cw) and the "momentum-driven wind" (vzw) models. The cw model assumes $v_{\text {wind }}=484 \mathrm{~km} \mathrm{~s}^{-1}$ and a mass loading factor $\eta=2$, while the vzw model uses the relations $v_{\text {wind }} \propto \sigma$ and $\eta \propto \sigma^{-1}$ ( $\sigma$ being the galaxy velocity dispersion).

Spectra were generated from the simulations using our software specexbin, which first calculates the physical properties along a line of sight and then applies ionization fractions from CLOUDY tables of an optically thin slab of gas with an impinging ionizing background field from Haardt \& Madau (2001) including quasars and star-forming galaxies. The resulting spectra were analyzed using the AutoVP package to generate Voigt-profile fits from which line statistics could be derived and the summed $\Omega(\mathrm{C}$ IV $)$ was calculated.

\section{Continual vs. Early Enrichment}

Figure 1 displays the evolution of $\Omega(\mathrm{C}$ IV $)$ for a variety of observations and our $\mathrm{cw}$ and vzw models. A comparison to $\Omega(\mathrm{C})$ indicates significant ionization cor- 


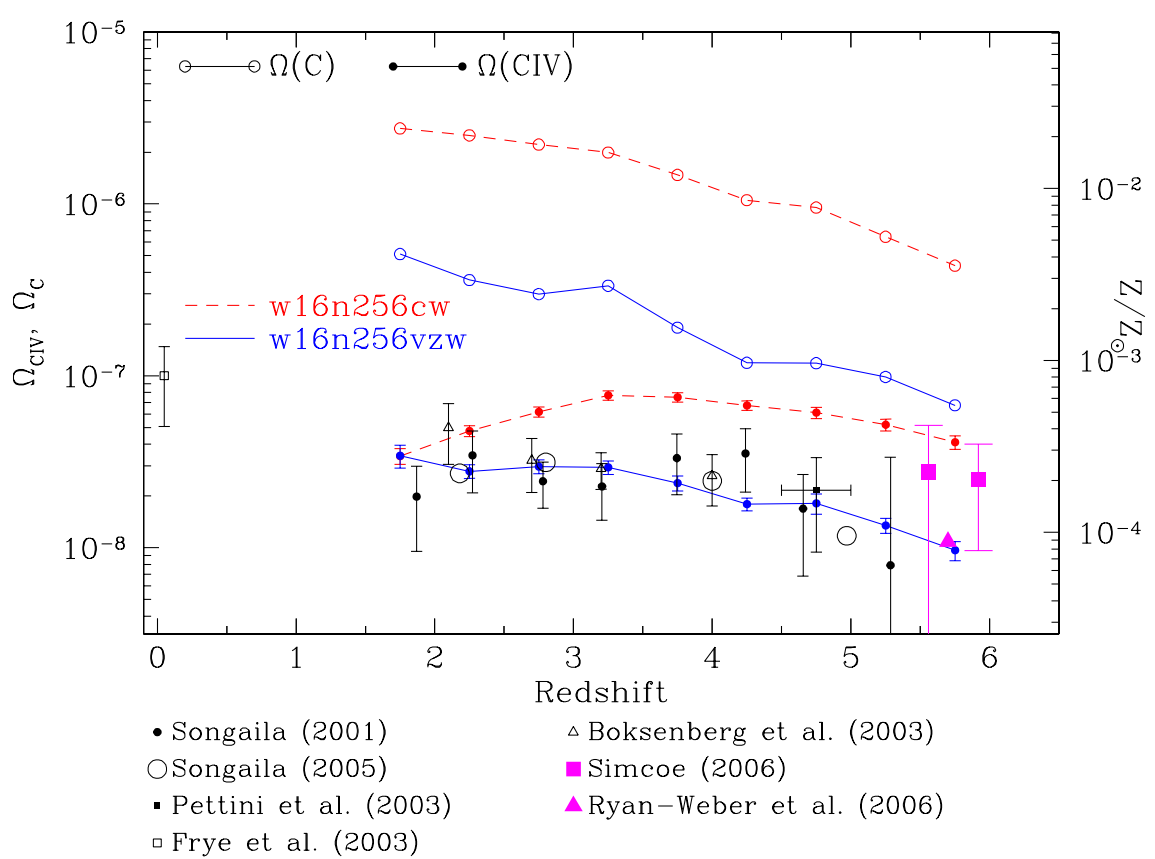

Fig. 1. Evolution of $\Omega(\mathrm{C}$ IV) and $\Omega(\mathrm{C})$, the mass density of $\mathrm{C}$ IV and metals, respectively. The constant wind (cw) model is overly efficient at enriching the IGM early, and creates an excessively hot IGM resulting in a much higher $\mathrm{C}$ IV ionization correction that increases too sharply at later times. The momentum-driven wind (vzw) injects fewer metals in the IGM, but does not require as extreme ionization correction due to less heating of the IGM from slower wind velocities at earlier times. The Ryan-Weber et al. data point is a lower limit.

rections, usually in excess of 10 are necessary to derive the true metallicity from $\Omega$ ( C IV). The ionization correction increases toward lower redshift for three main reasons. First and most importantly for the vzw model, the physical densities decrease at lower redshift meaning that C IV traces metals at higher overdensities, which occupy less volume at lower redshift. Second, the ionization background strengthens between $z=6 \rightarrow 2$ pushing the metals traced by CIV to slightly higher overdensity. Third, the metals become more shock-heated at later times due to metal-enriched winds colliding with increasingly faster infalling material toward low redshift. Metals are pushed into regions lower overdensity and higher temperature where carbon becomes ionized into $\mathrm{CV}$ and $\mathrm{C}$ VI. This third effect is greatest for the cw model causing its downturn in $\Omega(\mathrm{C}$ IV) at $z<3$.

New observations at $z>5.5$ (Ryan-Weber et al. 2006. Simcoe 2006) may suggest even more $\mathrm{C}$ IV at high redshift than our models produce. The momentumdriven galactic superwind feedback prescription based on observations of local 


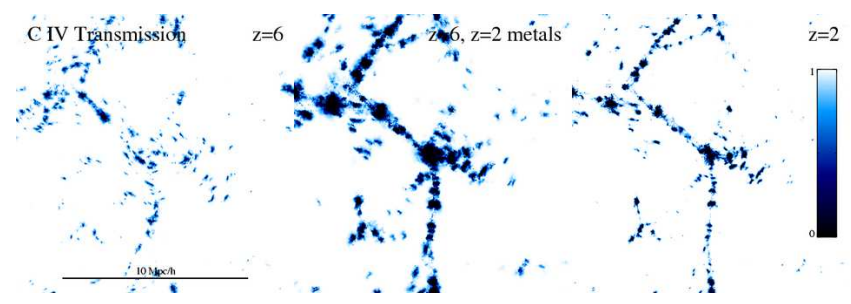

Fig. 2. These three panels show the CIV absorption in a $100 \mathrm{~km} \mathrm{~s}^{-1}$ slice of the vzw model $\left(15 h^{-1} \mathrm{Mpc}\right.$ across). The left and right panels show the metals at $z=6$ and $z=2$ respectively. The middle panel represents the early enrichment scenario where the metals in our simulation at $z=2$ are placed at $z=6$, thus assuming metals passively evolve from $z=6 \rightarrow 2$. The $\mathrm{C}$ IV ionization correction is much lower at $z=6$ resulting in $\sim 4 \times$ more absorption at $z=6$ and an $\Omega(\mathrm{C}$ IV) that must decline by a factor of at least two from $z=6 \rightarrow 2$, which is not observed. We can therefore rule out that the majority of metals were injected into the IGM before $z=6$.

starbursts and star formation rates may not be enough to enrich the high- $z$ IGM. We do not rule out the possibility of another form of metal enrichment from the earliest galaxies and stars, although we cannot conclude this definitely. Nevertheless, our point is to show that metal enrichment from continual star formation is required to produce most of the observed C IV by $z=2$.

As an exercise to show that the majority of metals cannot be injected into the IGM at early times, we take the metals in our simulation at $z=2$ and put them at $z=6$ densities and ionization conditions. This assumes that the metals passively evolve since $z=6$ without any new metals being injected. We also allow the IGM to cool to the $z=6$ IGM equation of state (EoS) at $\delta<100$, thus assuming that the metals were injected early enough to allow the IGM to cool. Slices of absorption in Figure 2 show how this case (middle) would result in much more absorption than the original $z=6$ or $z=2 \mathrm{vzw}$ case (left \& right respectively). The lower ionization corrections at $z=6$ result in $4 \times$ more absorption leading to a predicted drop in $\Omega(\mathrm{CIV})$ of at least $2 \times$. Even if we leave the metals at the $z=2$ shocked temperatures, there is still a significant decline. The fact that a constant $\Omega(\mathrm{C}$ IV $)$ is observed is strong evidence that metals in the IGM must be continually injected during the age of peak star formation.

Finally, we explore the age of the typical C IV absorber at $z=2$. We track the histories of individual SPH particles in our hydrodynamical simulation to see the redshift of their last injection into the IGM. Figure 3 plots the redshift histogram of a) the metal production (proportional to the global star formation rate), b) the last injection into the IGM of those metals, and c) the same as (b) but for metals traced by CIV. Although $\sim 40 \%$ of the gaseous metals are in galactic/halo gas, only $15 \%$ of C IV absorption in spectra comes from this gas leaving the other $85 \%$ arising from the IGM. The median age of a C IV absorber (ie. the last time it was in a wind) is 1 Gyr at $z=2$. CIV traces metals injected at a variety of ages, 


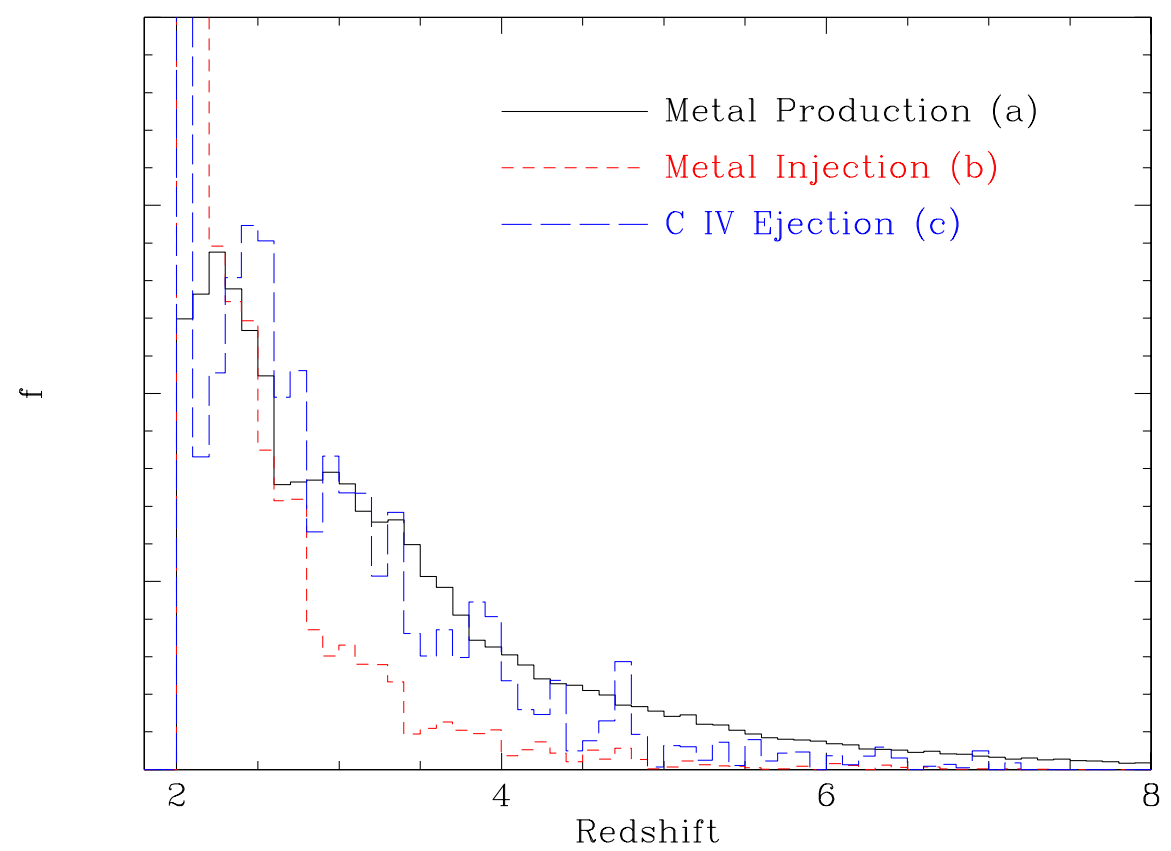

Fig. 3. The black histogram (a) represents the fraction of metals produced at that redshift (proportional to the global star formation rate) indicating that $95 \%$ of metals at $z=2$ are generated at $z<6$ in the vzw model. On the same scale, the red short-dashed histogram (b) is the redshift when the metals were last injected into the IGM via a wind, while the blue long-dashed histogram (c) is the same except for metals traced by C IV. $\sim 15 \%$ of $\mathrm{C}$ IV absorption at $z=2$ arises from galactic/halo gas, which accounts for $\sim 40 \%$ of the gaseous metals. The median C IV absorber traces metals injected $1 \mathrm{Gyr}$ ago at $z=2$. Thus C IV traces neither metals from only recent star formation nor early enrichment, but instead from a variety of ages. All histograms are on the same scale to allow one to compare them.

and does not exclusively trace metals from very recent star formation nor metals from early enrichment. The average particle is injected into the IGM via a wind more than twice by $z=2$, indicating there is significant recycling of metals in and out of the IGM. Older metals lie in lower overdensities suggesting observations that indicate C IV resides at a variety of overdensities (ie. the pixel optical depth method) require that metals be injected into the IGM at a variety of redshifts.

\section{Conclusions}

Our cosmological hydrodynamic simulations using momentum-driven winds from star-forming galaxies fit the main C IV observables between $z=6.0 \rightarrow 1.5$ as de- 
Title : will be set by the publisher

scribed in OD06. We further emphasize:

- The $\Omega(\mathrm{CIV})$ measurement remains relatively invariant after $z=6$ despite a vast majority $(>90 \%)$ of the IGM becoming enriched between $z=6 \rightarrow 2$. The ionization correction of $\mathrm{C}$ IV decreases due to 1) decreasing physical densities, 2) increasing ionization background strength, and 3) metals becoming more shockheated at lower redshift.

- We disfavor an early enrichment-only scenario, because it would lead to a decreasing $\Omega(\mathrm{C}$ IV $)$ with time due to the increasing $\mathrm{C}$ IV ionization correction. The IGM is primarily enriched via continual outflows from star-forming galaxies, although a different form of metal enrichment from the first stars/galaxies may be required for the $z>5.5 \mathrm{C}$ IV observations.

- CIV traces metals injected into the IGM at a variety of ages, with the median age of 1 Gyr at $z=2$. Only $15 \%$ of $\mathrm{CIV}$ arises from galactic/halo gas, while the other $85 \%$ is from the IGM. The spread of ages of IGM metals may be necessary to enrich the variety of overdensities as indicated by observations.

\section{References}

Adelberger, K. L., Steidel, C. C., Shapley, A. E., \& Pettini, M. 2003, ApJ, 584, 45

Adelberger K. L., Shapley A. E., Steidel C. C., Pettini M., Erb D. K., \& Reddy N. A. 2005, ApJ, 629, 636

Aguirre, A., Hernquist, L., Schaye, J., Katz, N., Weinberg, D. H., \& Gardner, J. 2001, ApJ, 561, 521

Boksenberg, A., Sargent, W. L. W., \& Rauch, M. 2003, ASP Conference Proceedings, Vol. 297, 447, eds. Edited E. Perez, R.M.G. Delgado, \& G. Tenorio-Tagle

Frye, B. L., Tripp, T. M., Bowen, D. B., Jenkins, E. B., \& Sembach, K. R. 2003, in "The IGM/Galaxy Connection: The Distribution of Baryons at $\mathrm{z}=0$ ", ASSL Conference Proceedings Vol. 281, 231, eds. J.L. Rosenberg \& M.E. Putman

Haardt, F. \& Madau, P. 2001, in "Clusters of galaxies and the high redshift universe observed in X-rays, Recent results of XMM-Newton and Chandra", XXXVIth Rencontres de Moriond, eds. D.M. Neumann \& J.T.T. Van.

Martin, C. L. 2005, ApJ, 621, 227

Murray, N., Quatert, E., \& Thompson, T. A. 2005, ApJ, 618, 569

Oppenheimer, B. D. \& Davé, R. 2006, MNRAS, accepted, astro-ph/0605651

Pettini M., Shapley A. E., Steidel C. C., Cuby J.-G., Dickinson M., Moorwood A. F. M., Adelberger K. L., \& Giavalisco M. 2001, ApJ, 554, 981

Pettini, M., Madau, P., Bolte, M., Prochaska, J.X., Ellison, S.L., \& Fan, X. 2003, ApJ, 594, 695

Ryan-Weber, E. V., Pettini, M., \& Madau, P. 2006, MNRAS, 371, 78

Rupke, D. S., Veilleux, S., \& Sanders, D. B. 2005, ApJS, 160, 115

Schaye, J., Aguirre, A., Kim, T.-S., Theuns, T., Rauch, M., \& Sargent, W.L.W. 2003, ApJ, 596,768

Simcoe, R. A. 2006, ApJ, submitted, astro-ph/0605710

Songaila, A. 2001, ApJ, 561, L153

Songaila, A. 2005, AJ, 130, 1996

Springel, V. 2005, MNRAS, 364, 1105 